\title{
Favorable Outcomes of Elderly ABO-Incompatible Kidney Transplantation-Pilot Single Center Experience
}

Kosoku A. Uchida J. Kabei K. Nishide S. Shimada H. Iwai T. Kuwabara N. Naganuma T. Maeda K. Kumada N. Takemoto Y. Nakatani T

\begin{tabular}{|c|l|}
\hline Citation & Urologia Internationalis, 101(4); 459-466 \\
\hline Issue Date & $2018-11$ \\
\hline Type & Journal Article \\
\hline Textversion & Author \\
\hline Rights & $\begin{array}{l}\text { This is the peer-reviewed but unedited manuscript version of the following article: } \\
\text { Urologia Internationalis } 2018 ; 101: 459-466 . \text { The final, published version is available } \\
\text { at } \underline{\text { https://doi.org/10.1159/000492721 }}\end{array}$ \\
\hline DOI & $10.1159 / 000492721$ \\
\hline
\end{tabular}

\author{
Self-Archiving by Author(s) \\ Placed on: Osaka City University
}


Title:

Favorable outcomes of elderly ABO-incompatible kidney transplantation-pilot single center experience

Authors:

Akihiro Kosoku ${ }^{1}$, Junji Uchida $^{1}$, Kazuya Kabei ${ }^{1}$, Shunji Nishide ${ }^{1}$, Hisao Shimada ${ }^{1}$, Tomoaki Iwai $^{1}$, Nobuyuki Kuwabara ${ }^{1}$, Toshihide Naganuma ${ }^{1}$, Keiko Maeda ${ }^{2}$, Norihiko Kumada ${ }^{3}$, Yoshiaki Takemoto ${ }^{1}$, Tatsuya Nakatani ${ }^{1}$

\section{Affiliation:}

${ }^{1}$ Department of Urology, Osaka City University Graduate School of Medicine.

${ }^{2}$ Department of Nursing, Osaka City University Hospital.

${ }^{3}$ Department of Urology, Suita Municipal Hospital.

\section{Short title:}

Elderly ABO-incompatible kidney transplantation

\section{Correspondence author:}

Junji Uchida, MD., PhD.

Department of Urology, Osaka City University Graduate School of Medicine

1-4-3, Asahi-machi, Abeno-ku, Osaka, 545-8585, Japan.

Phone: +81-6-6645-3857 Fax: +81-6-6647-4426

E-mail: m9492120@msic.med.osaka-cu.ac.jp 


\section{Abstract}

\section{Background}

The growth in the end-stage kidney disease population has been predominantly in the older adult population. In Japan, ABO-incompatible kidney transplantation has become an acceptable treatment option. However, few studies have been made on elderly ABO-incompatible kidney transplantation.

\section{Patients and Methods}

Seventeen patients aged 60 years and older who received their grafts from ABO-incompatible living donors at our institution between December 2006 and September 2016 were enrolled in this study, and the outcome of these recipients was evaluated.

\section{Results}

All 17 patients underwent successful kidney transplantation. Both overall patient and graft survival rates were $100 \%, 100 \%$, and $83.3 \%$ at posttransplant 1,3 , and 5 years, respectively. Six of the 17 patients $(35.3 \%)$ had an episode of biopsy-proven acute cellular rejection. Two patients who developed steroid- and deoxyspergualin-resistant acute rejection required anti-human thymocyte immunoglobulin.

\section{Conclusion}

ABO-incompatible kidney transplantation may be an effective radical renal replacement therapy for elderly patients with ESKD, although it could be a high-risk procedure.

Key words: kidney transplantation, ABO-incompatible, elderly 


\section{Introduction}

Kidney transplantation recipients, irrespective of their age, have a lower mortality rate compared to patients on dialysis [1]. Moreover, the elderly mostly account for the increase in patients with end-stage kidney disease (ESKD) [2]. The mean age at which patients are initiated to dialysis therapy stands at 69.2 years of age according to the 2015 data for Japan. Previous reports have demonstrated a significantly longer life expectancy among patients aged 60 years and older with deceased donor kidney transplantation compared to patients aged 60 years and older on the waiting list [1]. In Japan, owing to the severe shortage of deceased donors, living donor kidney transplantation is mainly performed, and the number of ABO-incompatible kidney transplantation is currently increasing. As a result, it has become an acceptable treatment option in Japan, accounting for more than $30 \%$ of all living donor kidney transplantation. Excellent outcomes have been achieved, and the rates of graft survival in these patients are presently similar to those in recipients of ABO-compatible grafts $[3][4][5][6][7]$. Although our previous report demonstrated that this procedure may become a viable treatment option for elderly patients with ESKD [8], there have been few reports on elderly ABO-incompatible kidney transplantation.

ABO-incompatible kidney transplantation is considered an immunologically high-risk procedure. Immunosuppressive regimens consist of administration of rituximab to inhibit antibody production, plasmapheresis to remove the anti-A/B antibodies, and pharmacotherapy [9][10][11][12][13]. Age-related changes occur in most organs and can alter the pharmacodynamics response. These changes could potentially affect drug responsiveness and result in altered toxicity or influence drug-drug interactions [14]. Previously, it has been 
reported that kidney transplant recipients over age 60 have diminished immune activity and low risk of rejection [15]. The immune system of the elderly undergoes immunosenescence, which protects the patient from rejection but increases the risk of infection and cancer [16]. In this tudy, we summarized our experience with $\mathrm{ABO}$-incompatible kidney transplantation in elderly patients more than 60 years of age.

\section{Patients and Methods}

This is a retrospective, observational study to examine the clinical outcome of elderly ABO-incompatible kidney transplant recipients, focusing on protocols, complications, and patient and graft survivals. A total of 56 patients underwent ABO-incompatible kidney transplantation at our institution between December 2006 and September 2016, of which 17 recipients aged 60 years and older were enrolled in this study. All of these patients were followed up until November 2017.

Immunosuppressive protocols (Table 2)

Immunosuppressive protocols have been improved over time, and we have used the optimal protocol available at the time. As our present standard desensitization protocol to remove anti-A/B antibodies for elderly $\mathrm{ABO}$-incompatible kidney transplant recipients, the patients underwent 1 to 8 sessions of plasmapheresis prior to kidney transplantation until the antibody titers were less than 1:16. The patients received a single dose of rituximab (150 $\mathrm{mg} / \mathrm{m}^{2}$ ) at 2 weeks prior to transplantation to inhibit antibody production. The pretransplant immunosuppressive protocol included 2 weeks (after January 2012) or 4 weeks (before December 2011) of mycophenolate mofetil (MMF) 0.5-1.0 g/day ( $\mathrm{n}=16)$ or everolimus 1.5 
g/day ( $\mathrm{n}=1)$ for B-cell lymphocyte suppression. Basic immunosuppression after transplantation consisted of basiliximab, steroid, calcineurin inhibitors (cyclosporine or tacrolimus), and MMF or everolimus (Figure 1). Our protocol was modified depending on age and anti-A/B antibody titers. In cases that seemed to be high risk, additional desensitization was performed to avoid antibody-mediated rejection (AMR) by anti-A/B antibodies. The patient with an anti-A/B antibody titer of 1:2048 received two doses of rituximab (150 $\mathrm{mg} / \mathrm{m}^{2}$ ) (case 1). The patient who experienced rebound of anti-A/B antibody titer to $1: 128$ after antibody removal on the day before kidney transplantation (case 9) and the patient with a high anti-A/B antibody titer (anti-A/B antibody titer of 1:2048) underwent splenectomy additionally (case 1). This study was approved by the Ethics Committee of Osaka City University Graduate School of Medicine (No. 3957). Opt-out consent was obtained instead of written informed consent. We provided patients with information explaining the proposed research plan (the purpose, required individual data, and duration of research) by means of an information website of our hospital and gave them the opportunity of opt-out. All the procedures were in accordance with the Helsinki Declaration of 2000 and the Declaration of Istanbul 2008.

For treatment of acute cellular rejection episodes, methylprednisolone was administered at a dose of $500 \mathrm{mg} /$ day for 3 days alone or in combination with deoxyspergualin $(5 \mathrm{mg} / \mathrm{kg} / \mathrm{day}: 5-7$ days). When resistance to these drugs was confirmed, anti-human thymocyte immunoglobulin was administered. For treatment of AMR, plasmapheresis was performed, and rituximab was administered. Acute cellular rejection and AMR was histologically diagnosed by light microscopy of graft biopsy specimens according 
to Banff 2007-2013 criteria.

Surveillance biopsies were performed once within a month after surgery and before discharge from hospital in all patients. When clinically indicated by rising serum creatinine or decreasing urine output, episode biopsies were performed.

Anti-A/B antibody titers were measured pre- and post-transplantation. The saline agglutination technique was used for the measurement of the anti-IgM titers. The indirect Coombs' test was used for the measurement of the anti-IgG titers.

All patients were examined for pre-formed donor specific antibody, using flow cytometry crossmatch, flow panel reactive antibody, or LABScreen single antigen beads before transplantation.

\section{Results}

\section{Patient characteristics}

The median age of the recipients and donors was 66 (62-74 years) and 65 (30-74 years), respectively. The median dialysis period was 29 months (0-98 months). The most frequent origin of ESKD was diabetes nephropathy $(n=5,29.4 \%)$. Nine recipients $(52.9 \%)$ enrolled in this study had experienced cardiovascular disease, such as coronary artery disease, stroke, and related vascular disease as pretransplant medical history. The median anti-A/B IgG and IgM titers were 1:16 (1:2-1:2048) and 1:16 (1:2-1:512), respectively. The median observation period was 32.0 months (range 14 months to 132 months). No patients had pre-formed donor specific antibodies before transplantation. The patient characteristics are given in Table 1.

Patient and graft survival (Table 3) 
All 17 patients underwent successful kidney transplantation. Both overall patient and graft survival rates were $100 \%, 100 \%$, and $83.3 \%$ at 1,3 , and 5 years, respectively (Figure 2). Death-censored graft survival rates were $100 \%$ at 5 years. One patient died 50 months after transplantation with a functioning graft due to chronic heart failure. The other 16 patients did not experience graft loss or death after transplantation and maintained good graft functions. The median serum creatinine level was $1.22 \mathrm{mg} / \mathrm{dl}(1.02-1.58 \mathrm{mg} / \mathrm{dl})$. The median observation period after transplantation was 44 months (range: 14 to 132 months).

\section{Rejection (Table 3)}

Six of the 17 patients (35.3\%) had an episode of biopsy-proven acute cellular rejection. Borderline changes with graft dysfunction were included as acute cellular rejection. One patient had acute cellular rejection classified as Banff IA, and 2 had borderline rejection with graft dysfunction, and they received steroid pulse therapy and/or administration of deoxyspergualin. One experienced acute rejection that was diagnosed with isolated V lesion and was treated with steroid pulse therapy in combination with administration of deoxyspergualin. Two patients who developed steroid- and deoxyspergualin-resistant acute rejection required anti-human thymocyte immunoglobulin. No patients experienced AMR.

\section{Complications (Table 3)}

Twelve patients experienced cytomegalovirus reactivation revealed by cytomegalovirus antigenemia. However, no obvious invasive tissue disease occurred. No patients experienced BK virus nephropathy. Two patients developed herpes zoster due to reactivation of varicella zoster virus and were treated with administration of acyclovir. One experienced adenovirus-induced cystitis. One patient had suspected pneumocystis pneumonia 
that was treated with administration of sulfamethoxazole/trimethoprim 80 days after transplantation. Ten patients experienced late-onset neutropenia that was suspected to be caused by rituximab administration. They recovered from neutropenia by administration of granulocyte-colony stimulating factor and/or MMF temporary discontinuation. One patient developed carcinoma of the body and tail of the pancreas 15 months after transplantation and was treated by distal pancreatectomy with splenectomy. However, he experienced recurrence of carcinoma of the pancreas and was treated with chemotherapy. Two recipients underwent postoperative diffuse pelvic hemorrhage that needed surgical intervention (Clavien Dingo classification IIIb) during the perioperative period.

\section{Discussion}

Kidney transplantation is the most preferred renal replacement therapy in patients who are 60 years and over with ESKD because of better longevity and quality of life compared with dialysis therapy. However, ABO-incompatible kidney transplantation is a high-risk procedure requiring additional desensitization for anti-A/B antibodies, such as suppression of antibody production and antibody removal, and its benefits for elderly patients has remained unknown. In this present study, although one patient died with a functioning graft at 50 months after transplantation and one experienced carcinoma of the pancreas, we demonstrated that the outcome of this procedure for elderly patients who were 60 years or older was excellent. Therefore, ABO-incompatible kidney transplantation may be an effective radical renal replacement therapy for elderly patients with ESKD as previously described $[12]$. 
It is generally assumed that aging is associated with a progressive decline in immune function. Age-related immune deficiency is more prominent in adaptive immunity than in innate immunity [17]. Previous reports have demonstrated that elderly transplant patients have lower acute rejection rates and increment of vulnerability to infections and malignancies [16]. In fact, in this study, the CMV antigenemia-positive rate was $70.6 \%$ and reactivation of varicella zoster virus occurred in two patients, while one underwent hemorrhagic cystitis due to adenovirus and one developed malignant tumor. However, 35.3\% of the recipients enrolled in this study experienced acute cellular rejection, and two had intractable rejection that required anti-human thymocyte immunoglobulin. Indeed, ABO-incompatible kidney transplantation is commonly known to be a high-risk procedure. The relationship between the donor and recipient was spousal in 15 out of our 17 cases. Several reports have shown that kidney transplantation between spouses has a higher incidence of acute rejection because of poor histocompatibility [11]. Moreover, kidneys from older donors are more likely to have senile tissue injuries due to hypertension, microvascular renal damage, and glomerulomegaly and facilitate immune recognition and increased immunogenicity [18]. Elderly ABO-incompatible kidney transplantation might therefore have high risk for acute rejection. It may be unnecessary to reduce the intensity of immunosuppression for these elderly patients.

The patient who experienced malignant tumor developed intractable rejection, and anti-human thymocyte immunoglobulin was used to treat steroid- and deoxyspergualin-resistant acute rejection. This intensive anti-rejection therapy may have caused the appearance and progression of cancer in this patient. It may be necessary to 
develop a tailored desensitization protocol for preventing infections and malignancies and avoiding rejection in elderly $\mathrm{ABO}$-incompatible kidney transplant recipients. As such, everolimus may be an appropriate immunosuppressant for elderly ABO-incompatible kidney transplantation [19].

There were 5 recipients with ESKD due to diabetes nephropathy in this study. The value of eGFR in the recipients with diabetes mellitus (DM) was similar to that in the recipients without DM. There were no differences in clinical outcomes such as CMV infection and rejection episodes between the recipients with and without DM. In addition, the graft and patient survivals in the recipients with DM were $100 \%$. Although outcomes of kidney transplant recipients with DM remain inferior compared with recipients without DM [20], there might have been no significant differences in the outcomes between our elderly ABO-incomparable kidney transplant recipients with and without DM, because it was a small-sized pilot study.

With advanced age, the prevalence and severity, comorbidities tend to increase. In a European survey on patients with a mean age of 61 years undergoing renal replacement therapy, $28 \%$ had DM, 23\% ischemic heart disease, and $11 \%$ malignancy [21]. The review from the United Network for Organ Sharing Standard Transplant Analysis and Research File showed that the four strongest predictors of recipient survival after transplantation were age, DM, angina, and waiting time on dialysis [22]. In our present study, 5 (29.4\%) patients had DM and 9 (52.9\%) had cardiovascular disease as comorbidity. One patient died 50 months after transplantation due to chronic heart failure after valvular heart disease surgery. It is necessary to carefully evaluate the comorbidity of elderly patients with ESKD prior to kidney 
transplantation, as these recipients may be more susceptible to developing worsened comorbidity directly or indirectly due to powerful immunosuppressive therapy.

Frailty is characterized as an increased vulnerability to stressors with a decline in the reserve and function of multiple physiologic systems. Limited compensatory mechanisms lead to functional impairment and adverse health outcomes, such as disability, falls, decreased mobility, hospitalization, and death [23]. Frailty is frequent in elderly patients, and the presence of chronic kidney disease can double the risk of frailty [24]. Previous reports have demonstrated that pre-transplant frailty increases the risk of delayed graft function, early hospital readmission after kidney transplantation, MMF intolerance, and mortality [25]. Elderly patients with ESKD may be more associated with frailty and have less tolerance for desensitization in $\mathrm{ABO}$-incompatible kidney transplantation compared to younger patients. In this study, graft survival at 1 year and 3 years was both $100 \%$, because adequate pre-transplant examination of the recipients was carefully done except for frailty. However, in future, the evaluation of frailty should be surveyed before kidney transplantation in elderly $\mathrm{ABO}$-incompatible kidney transplant recipients requiring powerful desensitization.

Unintentional low medication adherence is a real, complex problem for older patients receiving polypharmacy. In a recent report, there was non-adherence at one month after hospital discharge in $55 \%$ of non-transplant patients older than 65 years [26]. The number of drugs prescribed at discharge was reported to be related to medication non-adherence [27]. Transplant recipients must take a variety of drugs every day. Although elderly transplant recipients tend to be more compliant to therapy than younger adults, the 
rate of adherence to immunosuppressive and supportive therapy decreases as patient age increases [28]. Desensitization for $\mathrm{ABO}$-incompatible kidney transplantation requires removal of anti-A/B antibodies and suppression of antibody production before transplantation. However, postoperative immunosuppressive regimens for ABO-incompatible kidney transplantation are the same as those for ABO-compatible cases [5][11]. Because elderly recipients of ABO-incompatible kidney transplantation do not need to take more drugs compared to elderly recipients of ABO-compatible kidney transplantation, the risk of medication non-adherence is comparable between the two.

There might be limitations in this study, because it is a retrospective study and consists of a small sample size. However, there have been few reports on elderly ABO-incompatible kidney transplantation. We herein reported on our experience using this procedure on elderly patients with ESKD as a pilot study, although is it could be a high-risk procedure.

In conclusion, we demonstrated that our excellent outcome of ABO-incompatible kidney transplantation for elderly patients aged 60 years or older. Elderly ABO-incompatible kidney transplantation may be an effective radical renal replacement therapy, and further examination including frailty should be evaluated prior to transplantation for elderly kidney transplant candidates.

\section{Conflict of interest}

The authors declare no conflicts of interest. 


\section{References}

1: Wolfe RA, Ashby VB, Milford EL, Ojo AO, Ettenger RE, Agodoa LY, Held PJ, Port FK. Comparison of mortality in all patients on dialysis, patients on dialysis awaiting transplantation, and recipients of a first cadaveric transplant. $\mathrm{N}$ Engl $\mathrm{J}$ Med. $1999 ; 341: 1725-1730$.

2: Danovitch G, Savransky E. Challenges in the counseling and management of older kidney transplant candidates. Am J Kidney Dis. 2006;47:S86-97.

3: Takahashi K, Saito K, Takahara S, Okuyama A, Tanabe K, Toma H, Uchida K, Hasegawa A, Yoshimura N, Kamiryo Y; Japanese ABO-Incompatible Kidney Transplantation Committee. Excellent long-term outcome of ABO-incompatible living donor kidney transplantation in Japan. Am J Transplant. $2004 ; 4: 1089-1096$.

4: Zschiedrich S, Kramer-Zucker A, Jänigen B, Seidl M, Emmerich F, Pisarski P, Huber TB. An update on ABO-incompatible kidney transplantation. Transpl Int. 2015;28:387-397.

5: Uchida J, Kuwabara N, Machida Y, Iwai T, Naganuma T, Kumada N, Nakatani T. Excellent outcomes of ABO-incompatible kidney transplantation: a single-center experience. Transplant Proc. $2012 ; 44: 204-209$.

6: Uchida J, Machida Y, Iwai T, Kuwabara N, Iguchi T, Naganuma T, Kumada N, Kawashima $\mathrm{H}$, Nakatani T. Clinical outcome of ABO-incompatible living unrelated donor kidney transplantation. Urol Int. 2011;86:307-314.

7: Okumi M, Toki D, Nozaki T, Shimizu T, Shirakawa H, Omoto K, Inui M, Ishida H, Tanabe K. ABO-Incompatible Living Kidney Transplants: Evolution of Outcomes and Immunosuppressive Management. Am J Transplant. 2016;16:886-896. 
8: Uchida J, Iwai T, Machida Y, Kuwabara N, Kabei K, Murao M, Otoshi T, Naganuma T, Kumada N, Nakatani T. ABO-incompatible kidney transplantation in elderly patients over 60 years of age. Int Urol Nephrol. 2012;44:1563-1570.

9: Opelz G, Morath C, Süsal C, Tran TH, Zeier M, Döhler B. Three-year outcomes following 1420 ABO-incompatible living-donor kidney transplants performed after ABO antibody reduction: results from 101 centers. Transplantation. 2015;99:400-404.

10: Takahashi K, Saito K. ABO-incompatible kidney transplantation. Transplant Rev (Orlando). 2013;27:1-8.

11: Uchida J, Iwai T, Kabei K, Nishide S, Yamasaki T, Kuwabara N, Naganuma T, Kumada N, Takemoto Y, Nakatanti T. ABO-Incompatible Living Kidney Transplant Recipients from Spousal Donors Receiving Rituximab. Urol Int. 2016;97:457-465.

12: Uchida J, Machida Y, Iwai T, Naganuma T, Kitamoto K, Iguchi T, Maeda S, Kamada Y, Kuwabara N, Kim T, Nakatani T. Desensitization protocol in highly HLA-sensitized and ABO-incompatible high titer kidney transplantation. Transplant Proc. 2010;42:3998-4002.

13: Uchida J, Iwai T, Kato M, Machida Y, Naganuma T, Kumada N, Yoshimura R, Kawashima H, Kim T, Nakatani T. A novel approach to successful ABO-incompatible high-titer renal transplantation. Transplant Proc. 2008;40:2285-2288.

14: Kuypers DR. Immunotherapy in elderly transplant recipients: a guide to clinically significant drug interactions. Drugs Aging. 2009;26:715-737.

15: Martins PN, Pratschke J, Pascher A, Fritsche L, Frei U, Neuhaus P, Tullius SG. Age and immune response in organ transplantation. Transplantation. 2005;79:127-132.

16: Trouillhet I, Benito N, Cervera C, Rivas P, Cofán F, Almela M, Angeles Marcos M, Puig 
de la Bellacasa J, Pumarola T, Oppenheimer F, Moreno-Camacho A. Influence of age in renal transplant infections: cases and controls study. Transplantation. 2005;80:989-992.

17: Li P, Tian C, Ge N, Wang H, Liu L, Lou F, Björkholm M, Xu D. Premature senescence of $\mathrm{T}$ cells in long-term survivors of renal transplantation. Biochem Biophys Res Commun. 2011;407:599-604.

18: Heinbokel T, Hock K, Liu G, Edtinger K, Elkhal A, Tullius SG. Impact of immunosenescence on transplant outcome. Transpl Int. 2013;26:242-253.

19: Uchida J, Machida Y, Iwai T, Kuwabara N, Kabei K, Naganuma T, Kumada N,Kawashima H, Nakatani T. Conversion of stable ABO-incompatible kidney transplant recipients from mycophenolate mofetil with standard exposure calcineurin inhibitors (CNIs) to everolimus with very low exposure CNIs-a short-term pilot study. Clin Transplant. 2014;28:80-87.

20: Lim WH, Wong G, Pilmore HL, McDonald SP, Chadban SJ. Long-term outcomes of kidney transplantation in people with type 2 diabetes: a population cohort study. Lancet Diabetes Endocrinol. 2017;5:26-33.

21: Stel VS, van Dijk PC, van Manen JG, Dekker FW, Ansell D, Conte F, Kramar R, Leivestad T, Vela E, Briggs JD, Jager KJ. Prevalence of co-morbidity in different European RRT populations and its effect on access to renal transplantation. Nephrol Dial Transplant. $2005 ; 20: 2803-2811$.

22: Baskin-Bey ES, Kremers W, Nyberg SL. A recipient risk score for deceased donor renal allocation. Am J Kidney Dis. 2007;49:284-293.

23: Morley JE, Vellas B, van Kan GA, Anker SD, Bauer JM, Bernabei R, Cesari M, Chumlea 
WC, Doehner W, Evans J, Fried LP, Guralnik JM, Katz PR, Malmstrom TK, McCarter RJ, Gutierrez Robledo LM, Rockwood K, von Haehling S, Vandewoude MF, Walston J. Frailty consensus: a call to action. J Am Med Dir Assoc. 2013;14:392-397.

24: Anand S, Johansen KL, Kurella Tamura M. Aging and chronic kidney disease: the impact on physical function and cognition. J Gerontol A Biol Sci Med Sci. 2014;69:315-322.

25: McAdams-DeMarco MA, Ying H, Olorundare I, King EA, Haugen C, Buta B, Gross AL,Kalyani R, Desai NM, Dagher NN, Lonze BE, Montgomery RA, Bandeen-Roche K, Walston JD, Segev DL. Individual Frailty Components and Mortality in Kidney Transplant Recipients. Transplantation. 2017;101:2126-2132.

26: Pasina L, Brucato AL, Falcone C, Cucchi E, Bresciani A, Sottocorno M, Taddei GC, Casati M, Franchi C, Djade CD, Nobili A. Medication non-adherence among elderly patients newly discharged and receiving polypharmacy. Drugs Aging. 2014;31:283-289.

27: Claxton AJ, Cramer J, Pierce C. A systematic review of the associations between dose regimens and medication compliance. Clin Ther. 2001;23:1296-1310.

28: Chisholm-Burns MA, Kwong WJ, Mulloy LL, Spivey CA. Nonmodifiable characteristics associated with nonadherence to immunosuppressant therapy in renal transplant recipients. Am J Health Syst Pharm. 2008;65:1242-1247. 


\section{Figure Legends}

1. Immunosuppressive protocol for elderly $\mathrm{ABO}$-incompatible kidney transplantation.

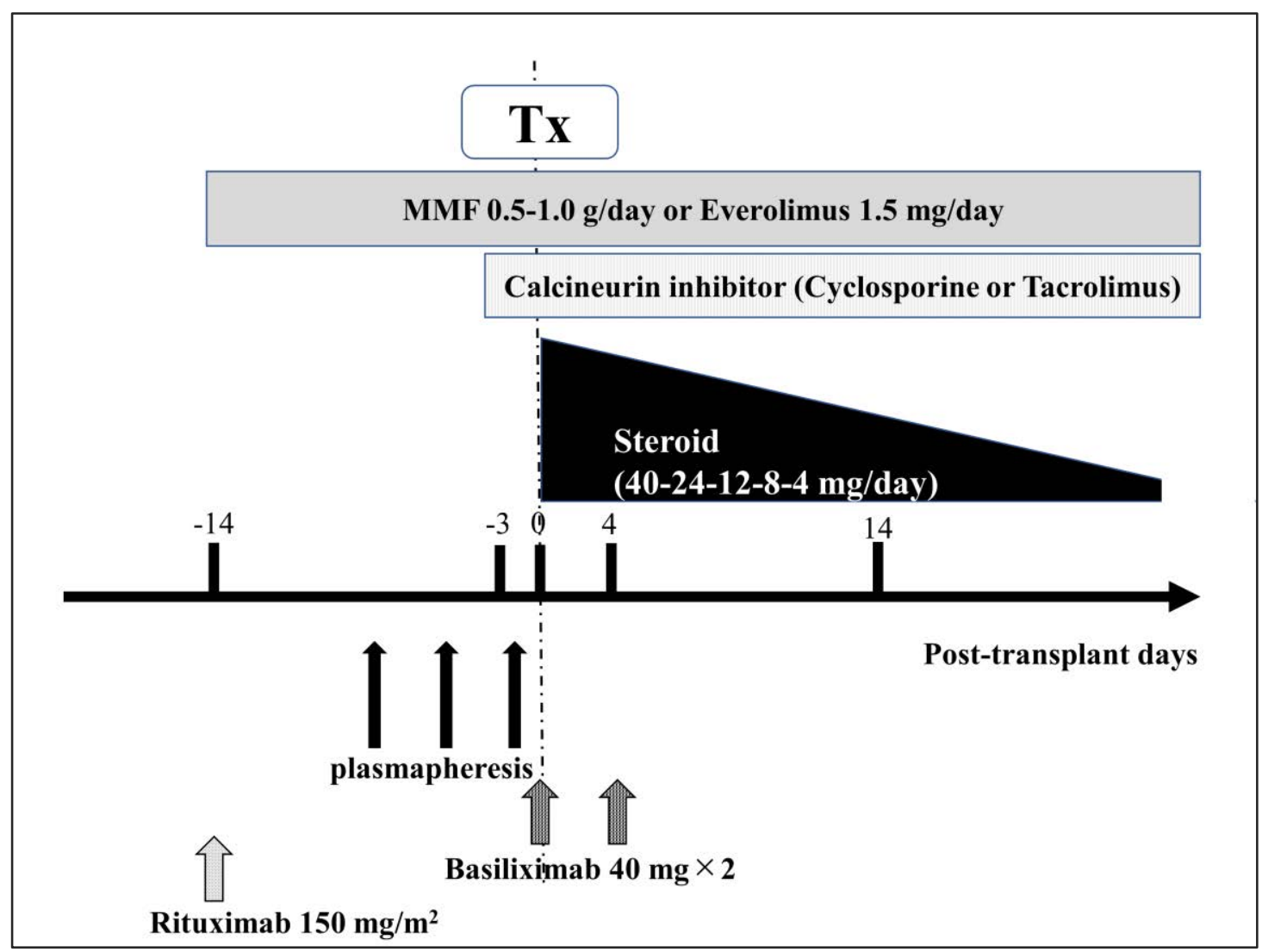


2. Kaplan-Meier curves for overall graft survival in elderly ABO-incompatible kidney transplantation.

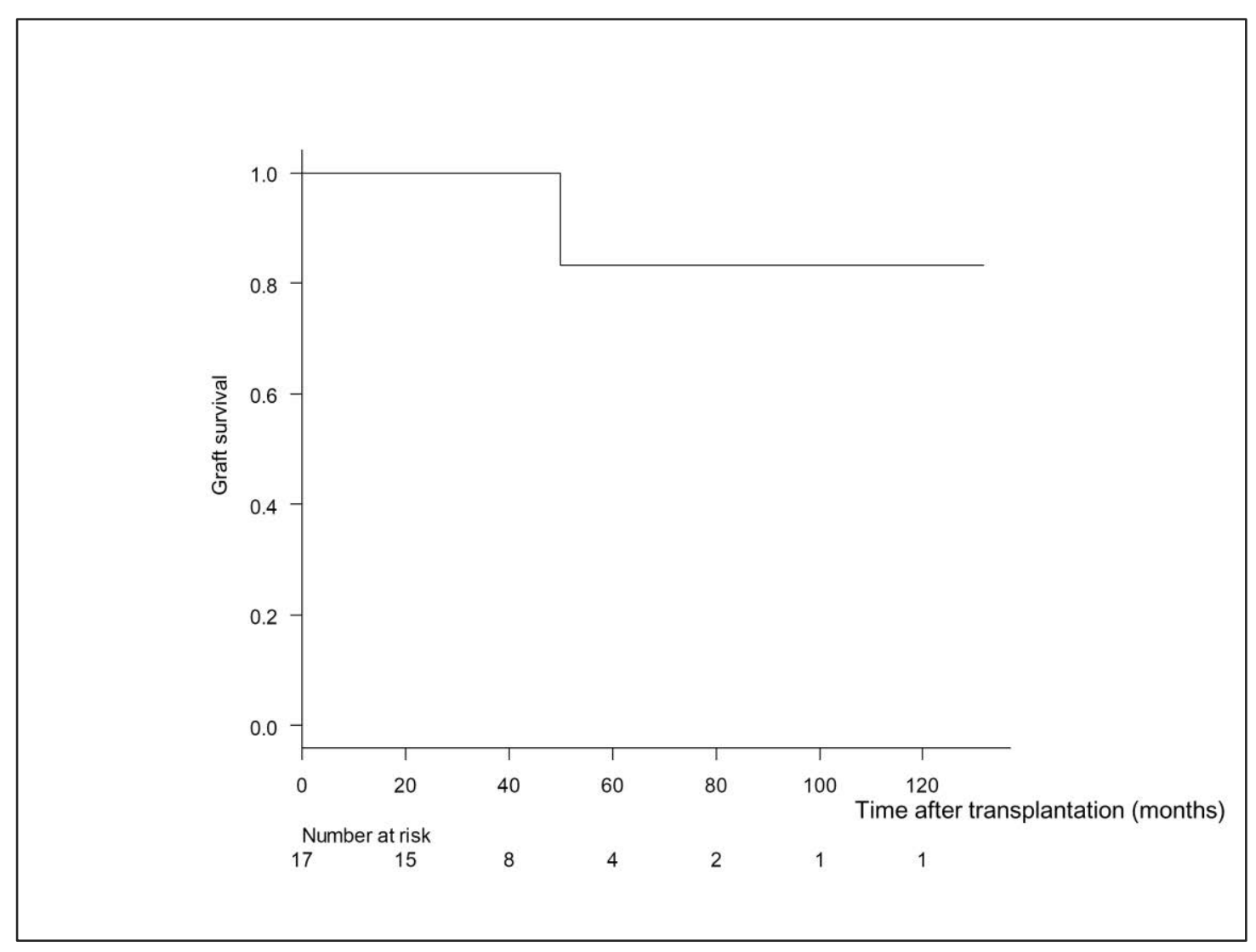




\begin{tabular}{|c|c|c|c|c|c|c|c|c|c|c|}
\hline Case & $\begin{array}{c}\text { Age at Tx } \\
\text { (year) }\end{array}$ & Gender & cause of ESKD & $\begin{array}{c}\text { donor age } \\
\text { (year) }\end{array}$ & donor & $\begin{array}{l}\text { dialysis } \\
\text { duration } \\
\text { (months) }\end{array}$ & $\begin{array}{c}\text { HLA } \\
\text { mismatch }\end{array}$ & Medical history & $\begin{array}{c}\text { Anti-A/B titer, } \\
\qquad \operatorname{IgG}\end{array}$ & $\begin{array}{c}\text { Anti-A/B titer, } \\
\text { IgM }\end{array}$ \\
\hline 1 & 64 & M & CGN & 65 & wife & 77 & 5 & & 2048 & 512 \\
\hline 2 & 74 & M & renal sclerosis & 50 & daughter & 8 & 3 & brain infarction & 2 & 2 \\
\hline 3 & 70 & M & unknown & 65 & wife & 40 & 3 & angina pectoris & 256 & 256 \\
\hline 4 & 69 & M & unknown & 66 & wife & 50 & 3 & & 32 & 32 \\
\hline 5 & 61 & M & ADPKD & 62 & wife & 53 & 5 & myocardia infarction & 8 & 8 \\
\hline 6 & 64 & $\mathrm{~F}$ & Lupus nephritis & 64 & husband & 29 & 4 & $\begin{array}{l}\text { myocardiac infarction, } \\
\text { brain infarction, } \\
\text { arteriosclerosis obliterans. }\end{array}$ & 16 & 16 \\
\hline 7 & 65 & M & $\begin{array}{l}\text { Membranous } \\
\text { nephropahy }\end{array}$ & 37 & wife & 0 & 4 & & 2 & 2 \\
\hline 8 & 66 & M & ADPKD & 66 & wife & 0 & 5 & & 8 & 8 \\
\hline 9 & 67 & M & unknown & 67 & wife & 0 & 4 & & 256 & 256 \\
\hline 10 & 64 & $\mathrm{~F}$ & DM nephropathy & 59 & husband & 9 & 4 & angina pectoris & 512 & 512 \\
\hline 11 & 69 & $\mathrm{~F}$ & DM nephropathy & 69 & husband & 9 & 3 & & 8 & 8 \\
\hline 12 & 67 & $\mathrm{~F}$ & ADPKD & 70 & husband & 98 & 4 & subarachnoid hemorrhage & 32 & 32 \\
\hline 13 & 66 & M & unknown & 66 & wife & 58 & 6 & rectal cancer, angina pectoris & 16 & 16 \\
\hline 14 & 65 & M & DM nephropathy & 63 & wife & 54 & 4 & $\begin{array}{l}\text { ureteral tumor (no malignancy), } \\
\text { ateriosclerosis obliterans, } \\
\text { myocardiac infarction }\end{array}$ & 64 & 64 \\
\hline 15 & 62 & M & DM nephropathy & 30 & son & 43 & 2 & angina pectoris & 8 & 8 \\
\hline 16 & 70 & $\mathrm{~F}$ & renal sclerosis & 73 & husband & 29 & 5 & breat cancer & 8 & 8 \\
\hline 17 & 66 & $\mathrm{M}$ & DM nephropathy & 74 & wife & 0 & 3 & hepatitis C & 64 & 64 \\
\hline
\end{tabular}

Tx, transplantation, ESKD; end-stage kidney disease, CGN; chronic glomerular nephritis, ADPKD; autosomal dominant polycystic kidney disease, DM; diabetes mellitus 
Table 2. immunosuppression

\begin{tabular}{lccccc}
\hline Case & $\begin{array}{c}\text { Dose of } \\
\text { rituximab }\end{array}$ & $\begin{array}{c}\text { Number of } \\
\text { plasmapheresis }\end{array}$ & $\begin{array}{c}\text { CNI } \\
(\text { CsA/Tac })\end{array}$ & MMF/Everolimus & Splenectomy \\
\hline 1 & 2 & 8 & Tac & MMF & + \\
2 & 1 & 3 & CsA & MMF & - \\
3 & 1 & 8 & CsA & MMF & - \\
4 & 1 & 4 & CsA & MMF & - \\
5 & 1 & 3 & Tac & MMF & - \\
6 & 1 & 2 & Tac & MMF & - \\
7 & 1 & 1 & CsA & MMF & - \\
8 & 1 & 2 & CsA & MMF & - \\
9 & 1 & 6 & Tac & MMF & + \\
10 & 1 & 3 & CsA & MMF & - \\
11 & 1 & 3 & CsA & MMF & - \\
12 & 1 & 3 & Tac & MMF & - \\
13 & 1 & 2 & Tac & MMF & - \\
14 & 1 & 5 & Tac & MMF & - \\
15 & 1 & 2 & CsA & MMF & - \\
16 & 1 & 2 & Tac & Everolimus & - \\
17 & 1 & 4 & Tac & MMF & - \\
\hline
\end{tabular}

CNI; calcineurin inhibitor, CsA; cyclosporine, Tac; tacrolimus,

MMF; mycophenolate mofetil. 
Table 3. Outcomes and complications

\begin{tabular}{|c|c|c|c|c|c|c|c|c|}
\hline Case & ACR & AMR & $\begin{array}{l}\text { Patient } \\
\text { survival }\end{array}$ & $\begin{array}{c}\text { Graft } \\
\text { survival }\end{array}$ & $\begin{array}{l}\text { Follow up period } \\
\qquad \text { (month) }\end{array}$ & $\begin{array}{l}\text { Current eGFR } \\
\left(\mathrm{ml} / \mathrm{min} / 1.73 \mathrm{~m}^{2}\right)\end{array}$ & $\begin{array}{c}\text { CMV } \\
\text { antigenemia }\end{array}$ & Other complication \\
\hline 1 & - & - & yes & yes & 132 & 54.85 & + & \\
\hline 2 & - & - & yes & yes & 85 & 49 & + & Postoperative hemorrhage \\
\hline 3 & - & - & no & no & 50 & & + & Pneumocystis pneumonia, Angina pectoris \\
\hline 4 & - & - & yes & yes & 77 & 37.63 & - & \\
\hline 5 & - & - & yes & yes & 63 & 46.15 & + & \\
\hline 6 & + & - & yes & yes & 53 & 35.25 & + & \\
\hline 7 & + & - & yes & yes & 43 & 42.11 & + & \\
\hline 8 & $+*$ & - & yes & yes & 41 & 47.15 & + & \\
\hline 9 & - & - & yes & yes & 32 & 38.48 & + & \\
\hline 10 & - & - & yes & yes & 31 & 42.96 & + & Passenger lymphocyte syndrome \\
\hline 11 & $+\#$ & - & yes & yes & 26 & 35.15 & + & Herpes zoster \\
\hline 12 & - & - & yes & yes & 25 & 40.06 & + & Adenovirus cystitis \\
\hline 13 & - & - & yes & yes & 21 & 40.41 & - & \\
\hline 14 & $+*$ & - & yes & yes & 20 & 40.07 & - & Carcinoma of the body and tail of the pancreas, Herpes zoster \\
\hline 15 & - & - & yes & yes & 20 & 35.21 & + & \\
\hline 16 & - & - & yes & yes & 15 & 45.15 & - & \\
\hline 17 & + & - & yes & yes & 14 & 33.4 & - & Postoperative hemorrhage \\
\hline
\end{tabular}

ACR ; acute cellular rejection, AMR; antibody-mediated rejection, eGFR; estimated glomerular filtration rate, CMV; cytomegalovirus. * steroid- and deoxyspergualin-resistant ACR,

\# ACR with isolated V lesion 